\title{
Cost and health impacts of adherence to the National Institute for Health and Care Excellence schizophrenia guideline recommendations
}

\author{
Huajie Jin, Paul Tappenden, James H. MacCabe, Stewart Robinson, Paul McCrone and Sarah Byford
}

\section{Background}

Discrepancies between the National Institute for Health and Care Excellence (NICE) schizophrenia guideline recommendations and current clinical practice in the UK have been reported.

\section{Aims}

We aim to assess whether it is cost-effective to improve adherence to the NICE schizophrenia guideline recommendations, compared with current practice.

\section{Method}

A previously developed whole-disease model for schizophrenia, using the discrete event simulation method, was adapted to assess the cost and health impacts of adherence to the NICE recommendations. Three scenarios to improve adherence to the clinical guidelines were modelled: universal provision of cognitive-behavioural therapy for patients at clinical high risk of psychosis, universal provision of family intervention for patients with first-episode psychosis and prompt provision of clozapine for patients with treatment-resistant schizophrenia. The primary outcomes were lifetime costs and quality-adjusted life-years gained.

\section{Results}

The results suggest full adherence to the guideline recommendations would decrease cost and improve quality-adjusted life-years. Based on the NICE willingness-to-pay threshold of
$£ 20000-£ 30000$ per quality-adjusted life-year gained, prompt provision of clozapine for patients with treatment-resistant schizophrenia results in the greatest net monetary benefit, followed by universal provision of cognitive-behavioural therapy for patients at clinical high risk of psychosis, and universal provision of family intervention for patients with first-episode psychosis.

\section{Conclusions}

Our results suggest that adherence to guideline recommendations would decrease cost and improve quality-adjusted lifeyears. Greater investment is needed to improve guideline adherence and therefore improve patient quality of life and realise potential cost savings.

\section{Keywords}

Guideline adherence; schizophrenia; economics; cognitivebehavioural therapy; clozapine.

\section{Copyright and usage}

(C) The Authors, 2020. Published by Cambridge University Press on behalf of the Royal College of Psychiatrists. This is an Open Access article, distributed under the terms of the creative commons Attribution licence (http://creativecommons.org/ licenses/by/4.0/), which permits unrestricted re-use, distribution, and reproduction in any medium, provided the original work is properly cited.
For people who are at risk of or diagnosed with schizophrenia, the key interventions recommended by the National Institute for Health and Care Excellence (NICE) include antipsychotic medications, cognitive-behavioural therapy (CBT) and family intervention. ${ }^{1}$ However, discrepancies between the NICE schizophrenia guideline recommendations and current clinical practice in the UK have been reported. A recent national audit ${ }^{2}$ suggested that CBT and family interventions were only offered to $<50 \%$ of eligible patients. It is suggested that this treatment gap might be largely related to underinvestment in services. ${ }^{3}$ In addition, there is evidence that despite the NICE schizophrenia guideline recommendation that clozapine should be offered to patients with treatmentresistant schizophrenia (TRS) with no delay, in clinical practice, there is a 4-year delay in providing clozapine to people with TRS. ${ }^{4}$ This might be caused by clinicians' concerns about clozapine-induced neutropenia and agranulocytosis, and the resources required for constant haematologic monitoring.

There is little evidence regarding the cost-effectiveness of practice guideline adherence for severe mental health disorders including schizophrenia. The closest example is a USA study that compared the clinical outcomes and healthcare costs between patients who received the Texas Medication algorithm-guided medication management or usual care, for patients with severe mental health disorders. ${ }^{5}$ However, the Texas Medication algorithm only covers pharmacological interventions and does not cover the full range of interventions available for these disorders. This study was therefore conducted in an effort to determine the cost-effectiveness of improved adherence to a number of recommendations from the NICE schizophrenia guideline, compared with current practice in the UK. Both pharmacological interventions (i.e. antipsychotic medications) and non-pharmacological interventions (e.g. CBT and family intervention) recommended by NICE were assessed.

A recent systematic review of existing economic models in schizophrenia found that $>80 \%$ of existing models assessed antipsychotic medications, whereas there were few models for non-pharmacological interventions. ${ }^{6}$ To our knowledge, this schizophrenia whole-disease model $(\mathrm{WDM})^{7}$ is the only model that covers both pharmacological and non-pharmacological interventions in the UK. In addition, the schizophrenia WDM was populated with high-quality input data and has been extensively checked and validated. Therefore, the schizophrenia WDM was used to simulate the impacts of guideline adherence in this study.

\section{Method}

The reporting of this study followed the recommendations of the Consolidated Health Economic Evaluation Reporting Standards statement. ${ }^{8}$ Per the Common Rule, ethical approval and informed patient consent were not required given that this is a modelling study with no direct patient contact or influence on patient care directly related to this work. Within the current study, the schizophrenia 
WDM was adapted to conduct a cost-utility analysis (CUA) to compare the cost-effectiveness of full adherence to the NICE schizophrenia guideline recommendations with current practice. CUA is one of the most widely used types of economic evaluation in healthcare. Within a CUA, effectiveness is measured in terms of generic 'healthy years', such as quality-adjusted life-years (QALYs). QALYs are generic measures of disease burden, which attempt to combine both the quality and the quantity of a person's life in response to a disease or a treatment for disease. One QALY is equivalent to a year in perfect health. To calculate QALYs, duration of life (survival) is weighted using 'utilities', which are estimated with appropriate preference-based measures of health-related quality of life (HRQoL). ${ }^{9}$ These measures of HRQoL are commonly anchored at 1 (perfect or best imaginable health) and 0 (death), with negative values possible in some scales to indicate a health state that is valued as less preferable than death. For example, if a patient has a utility of 0.5 over a period of 10 years, they would accrue 5 QALYs. As recommended by NICE, ${ }^{10}$ a costing perspective of the UK health and social care system was adopted.

\section{Model structure}

The schizophrenia WDM simulates the entire disease and treatment pathways for people at clinical high risk of psychoses (CHR) or with a diagnosis of psychosis or schizophrenia over a lifetime horizon. Patients enter the model following referral to secondary mental healthcare services for possible psychosis, and exit the model when they die. A discrete event simulation model was developed with SIMUL8 software (Simul8 Corporation, Glasgow, UK, professional version 15.0 for Windows; see https://www.simul8.com/online/). Discrete event simulation is a modelling method that simulates the experience of each individual patient. The clinical course of each patient is determined based on their characteristics, such as age, family history, previous events (e.g. relapses) and previous treatments.

The structure of the schizophrenia WDM is presented in Fig. 1. There are four modules within the WDM: specialist assessment pathway (module A), CHR pathway (module B), psychosis pathway (module C) and out-of-scope and death pathway (module D).

After entering the model through module A, patients are triaged to different modules based on the assessment results: CHR (module B), psychosis (module C) or not at risk of psychosis (module D). Patients at module B can move to module D (if they die) or module C (if they develop psychosis). Patients at module C cannot move to module $\mathrm{B}$, but they can move to module $\mathrm{D}$ if they receive a non-schizophrenia diagnosis or die. Patients at module D cannot move to other modules. Within each module, the intervention received by specific patients depends on three factors: their disease status (not at risk of psychosis, CHR, first-episode psychosis (FEP), subsequent acute psychosis or subsequent non-acute psychosis), availability of the intervention and patients' acceptance of non-compulsory interventions. In the model, it is assumed that patients may refuse to take up CBT and/or family intervention. The prescription of antipsychotic medications is assumed to be optional for patients with FEP in a stable condition, and is compulsory for all the other patients with psychosis. However, patients might discontinue their prescribed antipsychotic medications for various reasons. In the WDM, discontinuation of antipsychotic medications was modelled, and the primary reason for discontinuing antipsychotic medication were grouped into three mutually exclusive categories: inefficacy, intolerance and non-adherence not otherwise specified. Patients who discontinue their antipsychotic medication because of inefficacy are assumed to experience a relapse, and will be switched to a different antipsychotic medication after their relapses have been managed. Patients who discontinue their antipsychotic medication because of intolerance will be switched to a different antipsychotic medication without experiencing a relapse. Patients who discontinue antipsychotic medication because of non-adherence will no longer take any antipsychotic medication until they experience relapses. After managing relapse, those patients will either return to their previous antipsychotic medication, if this is the first time that they discontinue an oral antipsychotic medication because of non-adherence, or be switched to a long-acting injectable antipsychotic medication, if they have discontinued oral antipsychotic medications more than once. A comprehensive description of the model structure is reported by Jin et al.

\section{Prioritisation of interventions for modelling}

The NICE schizophrenia guideline recommended nine key interventions, including specialist mental health assessment, CBT, family intervention, antipsychotic medication, rapid triage assessment of acute psychosis by the crisis resolution and home treatment team, rapid tranquilisation, monitoring and treatment for coexisting conditions, and occupational and educational interventions. ${ }^{1}$ Because of data availability issues and resource constraints to this study, it was not possible to model adherence to all interventions recommended by the NICE schizophrenia guideline. Based on importance and data availability, three interventions were prioritised for evaluation with the model: individual CBT for people at CHR, family interventions for patients with FEP and oral clozapine for patients with TRS. The most common reason for excluding an intervention from this analysis was lack of evidence about the clinical and cost impacts of the intervention. A list of interventions excluded are listed in Supplementary File 1, Section 1 available at https://doi.org/10.1192/bjp.2020.241

\section{Key impacts of the interventions modelled in the study}

For each intervention under assessment, the key impacts modelled in the study, including clinical benefits, clinical harms, cost and cost savings, are reported in Table 1 . The impacts modelled in the study were chosen based on importance and data availability, as informed by published literature and expert opinion. Because of lack of evidence about the adverse events of CBT and family interventions, clinical harms were only modelled for antipsychotic medication. Five adverse events were modelled for antipsychotic medications: glucose intolerance, diabetes, weight gain, acute extrapyramidal symptoms and neutropenia.

\section{Input data}

Four types of input data were used in our study: clinical evidence, HRQoL, unit cost and healthcare resource use. A list of input data for the schizophrenia WDM is reported in a previous publication. ${ }^{\text {? }}$ The key parameters that are most relevant to this study are reported in Supplementary Table 2; these are briefly summarised below. The current provision of $\mathrm{CBT}$ and family intervention were obtained from the Early intervention in Psychosis national audit, ${ }^{2}$ which analysed data submitted by 55 mental health providers in England. A recent systematic review ${ }^{11}$ identified two studies that reported duration of delay in initiating clozapine for patients with TRS in the UK. The reported duration of delay ranges from 3.98 years in Howes et $\mathrm{al}^{4}$ to 5 years in Najim et al. ${ }^{12}$ The result reported by Howes et al was used in our study, because Howes et al was conducted more recently than Najim et al, and has a larger sample size. ${ }^{4}$ The clinical efficacy data of CBT, family intervention and CBT were informed by published meta-analyses. ${ }^{1,13,14}$ HRQoL data were obtained from published systematic reviews. ${ }^{1,15,16}$ Resource quantities were mainly informed by the systematic reviews conducted by the NICE schizophrenia guideline, ${ }^{17}$ new evidence identified from rapid literature review conducted for this study and expert opinion 


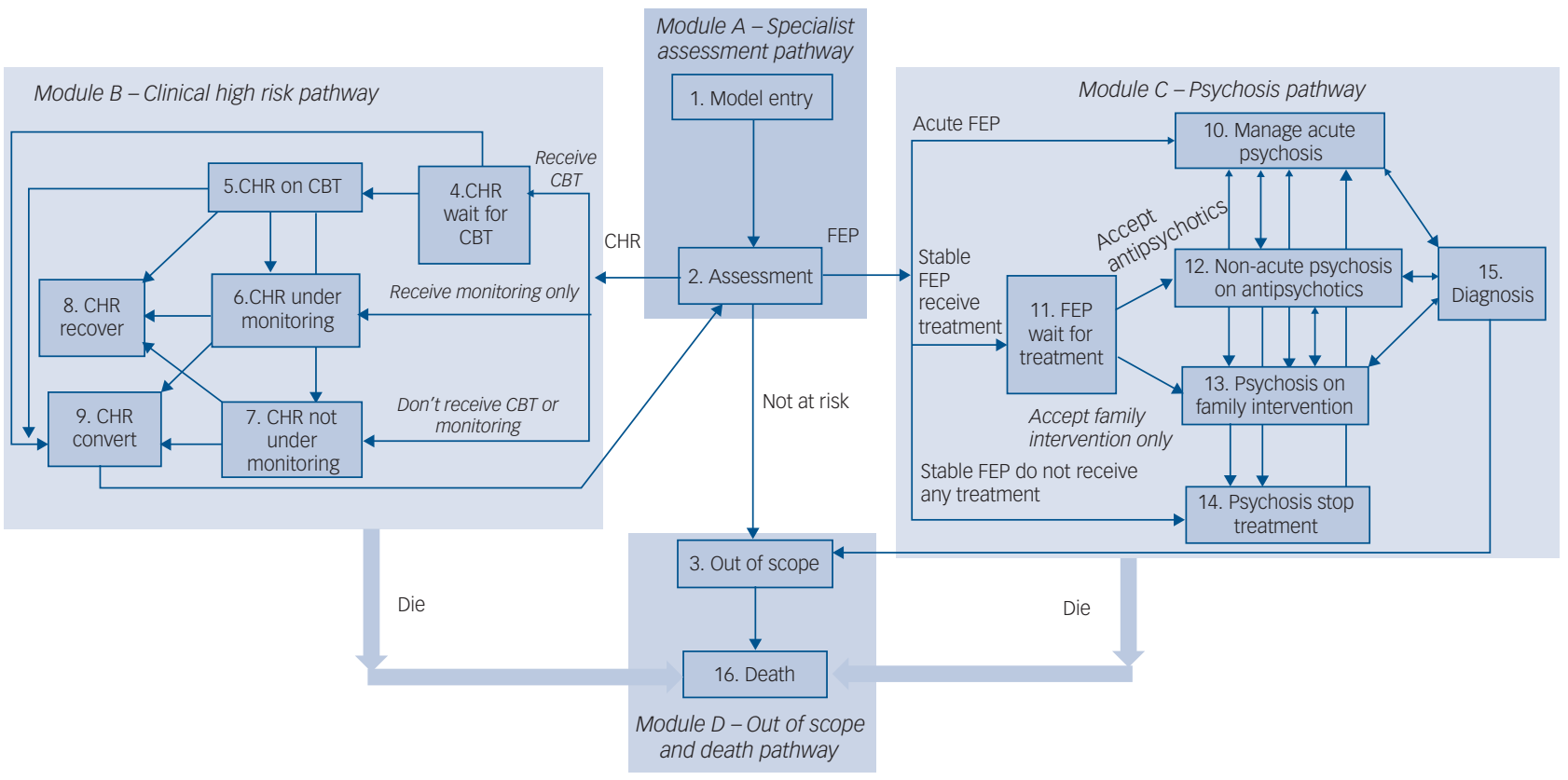

Fig. 1 Model structure. CBT, cognitive-behavioural therapy; CHR, clinical high risk of psychosis; FEP, first-episode psychosis.

where no evidence was identified. All costs were reported in 2016/ 2017 UK Pound Sterling. Unit costs were obtained from the Unit Costs of Health and Social Care 2017, ${ }^{18}$ prescription cost analysis (England) $2017^{19}$ and the NHS reference costs 2016-2017. ${ }^{20}$

\section{Outcomes}

The model estimates lifetime costs and lifetime QALYs gained for patients referred to secondary care mental health services for possible psychosis. An annual discount rate of 3.5\% was applied to both costs and QALYs. ${ }^{10}$ To facilitate comparison between different options, incremental cost-effectiveness ratios (ICERs) and incremental net monetary benefit (NMB) were calculated by using the estimates of costs and QALYs for each option assessed. The ICER is calculated as the difference in cost between two competing options, divided by the difference in QALYs. Cost-effectiveness is usually assessed by comparing an ICER against a reference threshold, which is intended to reflect the opportunity cost of displacing one QALY elsewhere in the health system in order to fund the new intervention. If the ICER falls below that threshold, the intervention might be considered to represent a good use of health resources, whereas if the ICER is above the threshold, the intervention is likely to be considered cost-ineffective. NICE's usual threshold $(\lambda)$ range for determining cost-effectiveness is $£ 20000-£ 30000$ per QALY gained for all interventions, except for life-extending treatments for people who are at the end of their life (defined as $<24$ months to live with current treatment) or for people with very rare diseases. Incremental NMB is a transformation of the ICER given some value of $\lambda$, which thereby reflects the monetary value of any additional QALYs gained, less any additional costs (calculated as $\Delta \mathrm{QALY} \times \lambda-\Delta$ Cost).

\section{Sensitivity analysis}

Three different types of sensitivity analyses were conducted to test the robustness of our results under different input data and/or assumptions: one-way sensitivity analysis, which assesses the impact of changing the value of individual parameter; structural sensitivity analysis, which assesses the impact of using alternative assumptions of the model (e.g. whether CBT can prevent psychosis or whether its effect is limited to delaying the transition from CHR to psychosis); and probabilistic sensitivity analysis (PSA), which assesses the joint impact of changing multiple input data simultaneously. In a PSA, each uncertain parameter is represented using a pre-defined probability distribution. Using Monte-Carlo sampling methods, each model run draws a random sample from each uncertain parameter distribution. For this study, the process was repeated 5000 times, resulting in a distribution of cost and health outputs.

\section{Results}

The results of the base-case analysis are reported in Table 2 and graphically presented in Fig. 2. As it shows in Table 2 and Fig. 2

\begin{tabular}{|c|c|c|c|c|}
\hline Interventions & Clinical benefits & Clinical harms & Costs & Cost savings \\
\hline CBT for people at CHR & $\begin{array}{l}\text { Delaying transition } \\
\text { from } \mathrm{CHR} \text { to } \\
\text { psychosis }\end{array}$ & None & Cost of providing CBT & $\begin{array}{l}\text { Delaying the } \\
\text { treatment costs of } \\
\text { psychosis }\end{array}$ \\
\hline $\begin{array}{l}\text { Family intervention for } \\
\text { people with FEP }\end{array}$ & $\begin{array}{l}\text { Preventing relapse of } \\
\text { psychosis }\end{array}$ & None & $\begin{array}{l}\text { Cost of providing family } \\
\text { intervention }\end{array}$ & $\begin{array}{l}\text { Reducing the cost of } \\
\text { treating relapse }\end{array}$ \\
\hline $\begin{array}{l}\text { Clozapine for people } \\
\text { with TRS }\end{array}$ & $\begin{array}{l}\text { Preventing relapse of } \\
\text { psychosis }\end{array}$ & $\begin{array}{l}\text { Adverse events, including acute EPS, weight } \\
\text { gain, glucose intolerance, diabetes and } \\
\text { neutropenia }\end{array}$ & $\begin{array}{l}\text { Cost of antipsychotic medication } \\
\text { and cost of treating adverse } \\
\text { events }\end{array}$ & $\begin{array}{l}\text { Reduced costs of } \\
\text { treating relapse }\end{array}$ \\
\hline
\end{tabular}




\begin{tabular}{|c|c|c|c|c|c|c|}
\hline Intervention & Cost, $f$ QALY & $\begin{array}{l}\text { Incremental } \\
\text { cost }\end{array}$ & $\begin{array}{l}\text { Incremental } \\
\text { QALY }\end{array}$ & ICER & $\begin{array}{c}\text { Ranking of NMB } \\
\text { (WTP }=20000 \text { per QALY) }\end{array}$ & $\begin{array}{c}\text { Ranking of NMB } \\
\text { (WTP }=30000 \text { per QALY) }\end{array}$ \\
\hline \multicolumn{7}{|l|}{ CBT for people at CHR } \\
\hline CBT (41.01\% availability) & 16807819.1904 & - & - & Dominated & 2 & 2 \\
\hline CBT (100.00\% availability) & 16745219.1904 & -626 & 0.0000 & Dominating & 1 & 1 \\
\hline \multicolumn{7}{|l|}{ Family intervention for people with FEP } \\
\hline Family intervention (30.98\% availability) & 16807819.1904 & - & - & Dominated & 2 & 2 \\
\hline Family intervention (100.00\% availability) & 16790519.2033 & -173 & 0.0129 & Dominating & 1 & 1 \\
\hline \multicolumn{7}{|c|}{ Clozapine for people with TRS } \\
\hline Clozapine (3.98-year delay) & 16807819.1904 & - & - & Dominated & 2 & 2 \\
\hline Clozapine (no delay) & 16221519.1977 & -4486 & 0.0052 & Dominating & 1 & 1 \\
\hline
\end{tabular}

(a) and 2(b), full adherence to the NICE schizophrenia guideline recommendations, for all three scenarios addressed, results in cost savings and gains in QALYs. Of the three scenarios addressed, universal provision of family intervention for patients with FEP produces the largest gain in QALYs (0.0129 QALY per person), whereas prompt provision of clozapine for patients with TRS produces the largest amount of cost savings ( $£ 4486$ per person). Fig. 2(c) presents the NMB of adherence to the NICE schizophrenia guideline recommendations for all three scenarios addressed. For both lower and higher thresholds of NICE ( $£ 20000$ and $£ 30000$ per QALY, respectively), prompt provision of clozapine for patients with TRS results in the greatest NMB, followed by universal provision of CBT for patients at CHR, and universal provision of family intervention for patients with FEP.

The base-case conclusion for provision of CBT and clozapine are robust to all one-way and structural sensitivity analyses

(a)

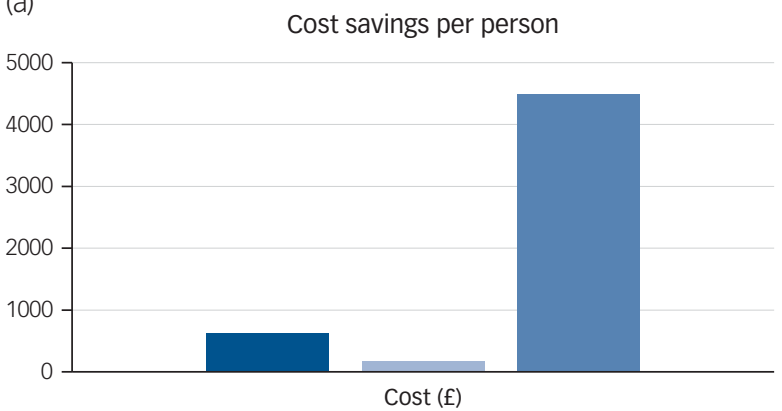

- Topic A. Universal provision of CBT for patients at CHR

- Topic B. Universal provision of family intervention for patients with an FEP

- Topic C. Prompt provision of clozapine for patients with TRS

(c)

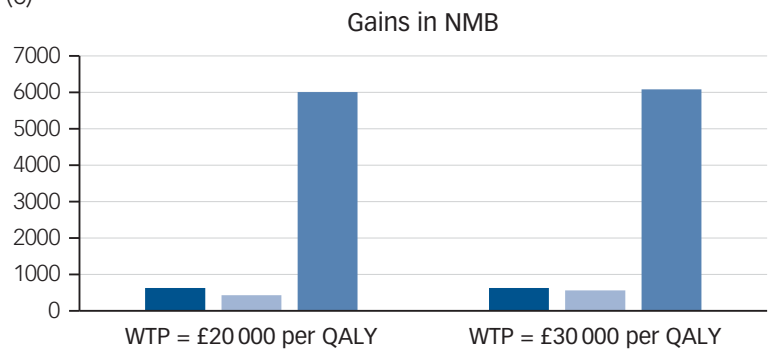

- Topic A. Universal provision of CBT for patients at CHR

- Topic B. Universal provision of family intervention for patients with an FEP - Topic C. Prompt provision of clozapine for patients with TRS conducted (Supplementary File 1, Section 3). The base-case conclusion for provision of family intervention is sensitive to the relative risk of family intervention in delaying relapse of psychosis. When the relative risk is increased from 0.63 to 0.83 , increasing the provision of family intervention from 30.98 to $100 \%$ results in increased costs and additional health gains, resulting in an ICER of $£ 160313$ per QALY gained.

The PSA results are consistent with the base-case results: at a $£ 20000$ per QALY threshold, the probability of being cost-effective compared with current practice is 0.74 for universal provision of CBT, 0.65 for universal provision of family intervention and 0.96 for prompt provision of clozapine; and at a $£ 30000$ per QALY threshold, the probability of being cost-effective compared with current practice is 0.75 for universal provision of CBT, 0.69 for universal provision of family intervention and 0.96 for prompt provision of clozapine.

(b)

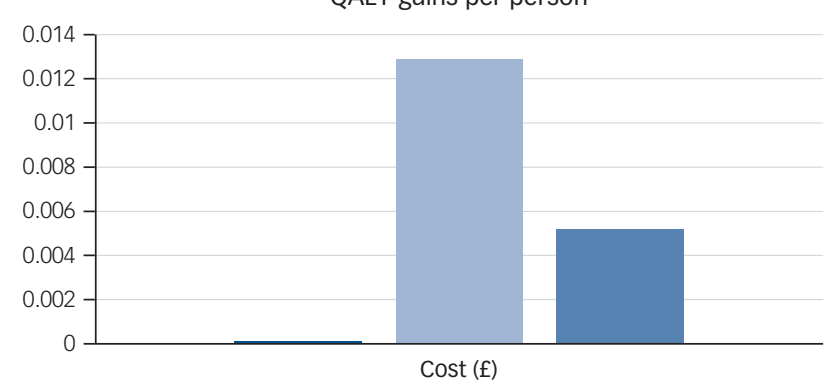

- Topic A. Universal provision of CBT for patients at CHR

Topic B. Universal provision of family intervention for patients with an FEP - Topic C. Prompt provision of clozapine for patients with TRS

Fig. 2 Net monetary benefit of adherence to topics A, B and C (per person). CBT, cognitive-behavioural therapy; CHR, clinical high risk of psychosis; FEP, first-episode psychosis; NMB, net monetary benefit; QALY, quality-adjusted life-years; TRS, treatment-resistant schizophrenia; WTP, willingness to pay. 


\section{Discussion}

\section{Main findings and interpretation}

The findings of this study suggest that adherence to the following NICE recommendations are likely to decrease cost and improve QALYs compared with the current practice, including: universal provision of CBT to people at CHR, universal provision of family intervention to people with FEP and prompt provision of clozapine to people with TRS.

This is likely to be because the cost of adherence to the NICE guideline recommendations (i.e. the cost of increasing provision of CBT, family intervention and monitoring patients on clozapine) is offset by the cost savings (i.e. delayed treatment cost of psychosis because of use of CBT for people at CHR, reduced cost of treating relapse for people with FEP, and reduced cost of treating relapse for people with TRS because of use of clozapine).

Of the three scenarios addressed, increasing the provision of family intervention from 30.98 to $100 \%$ produces the largest gain in QALYs. This might be because, compared with the other two scenarios, this intervention affects more patients. The target population of the schizophrenia WDM relates to people referred to secondary care mental health services for possible psychosis. Of the target population, the patient subgroup that can potentially benefit from each scenario and its proportion in the target population (based on the simulated outputs) is as follows: people at CHR and who accept CBT (17.80\%), people with FEP and who accept family intervention (41.17\%) and people with TRS (9.88\%).

These results suggest that the proportion of the population that can potentially benefit from universal provision of family intervention is much larger than for the other two scenarios, which may explain why adherence to the NICE recommendation for provision of family intervention results in the greatest gains in QALYs. In terms of costs, prompt provision of clozapine for patients with TRS results in the greatest cost savings. This might be because this scenario affects people with TRS and the cost of managing people with TRS is 3 to 11 times higher than people with schizophrenia who are not treatment resistant. ${ }^{21}$ Similarly, prompt provision of clozapine for patients with TRS results in the greatest NMB, followed by universal provision of CBT for patients at CHR, and universal provision of family intervention for patients with FEP.

To our knowledge, no similar analyses have been published that compare the cost and health outcomes of different levels of implementation of the NICE schizophrenia guideline recommendations. Therefore, it was not possible to compare the results of our analysis with published literature. However, previous published economic evaluation suggested that all three interventions assessed (CBT, family intervention and clozapine) are cost-effective for people at CHR or with a diagnosis of psychosis or schizophrenia. ${ }^{7}$ Therefore, increasing the provision or reducing delay in initiation of those interventions are likely to decrease cost and improve QALYs.

\section{Implications for clinical practice}

In the UK, although mental health disorders account for nearly a quarter of the total disease burden, mental health services only receives $13 \%$ of the total National Health Service budget. ${ }^{22}$ Further reductions in investments on mental health services have been reported in recent years, ${ }^{23}$ which may compromise service quality and affect patients' health outcomes. ${ }^{24}$ The results of this study show that one potential way to address the funding shortage without compromising patient outcomes is to improve adherence to the NICE schizophrenia guideline recommendations, as adherence to all three recommendations addressed results in additional health gains and cost savings. At the NICE willingness-to-pay thresholds of $£ 20000$ to 30000 QALY gained, the areas prioritised for implementations are, in order of importance: prompt provision of clozapine to people with TRS, universal provision of CBT for people at CHR and universal provision of family intervention to people with FEP.

\section{Strengths}

Our study has five strengths. First, this study presents the first analysis that assesses cost and health outcomes of adherence to good practice guidelines for schizophrenia. The results of this analysis can help commissioners and service providers to identity ways to improve outcomes while working within existing budgets. Second, while the existing economic evidence only assessed the impacts of adherence to one specific type of intervention (i.e. antipsychotic medication), ${ }^{5}$ this study assessed the impacts of adherence to three interventions within one analysis. The results of this study can be used to directly compare the potential cost savings and health benefits of improving implementation for different interventions simultaneously, and thus help stakeholders to prioritise areas of implementation when resources are scarce. Third, to prevent overestimation of the benefits of adherence to the NICE guideline recommendations, patient's acceptance and adherence to interventions were explicitly modelled. Thus, it was acknowledged that even when CBT and family intervention are provided, they might not be taken up by patients, and that people with TRS who are prescribed with clozapine might discontinue clozapine for various reasons. Fourth, the cost and health impacts of adverse events of antipsychotic medication were explicitly modelled. Five adverse events were modelled, including acute extrapyramidal symptoms, weight gain, glucose intolerance, diabetes and neutropenia. Finally, the stability of our results under different assumptions and different combinations of input data were tested with sensitivity analyses.

\section{Limitations}

There are four limitations of this study. First, of the nine interventions recommend by the NICE schizophrenia guideline, only three were assessed within this study. The six interventions that were not modelled were specialist mental health assessment, rapid triage assessment of acute psychosis by the crisis resolution and home treatment team, rapid tranquilisation, monitoring and treatment for coexisting conditions, and occupational and educational interventions. The most common reason for excluding an intervention from this analysis is lack of evidence about the clinical and cost impacts of the intervention. Second, although the results of this analysis suggest that adherence to the NICE schizophrenia guideline may reduce cost in the long term, the costs of improving adherence are often incurred earlier, whereas expected cost savings may take several years to be fully realised. This means at the early phases of improving adherence to the NICE guideline recommendations, commissioners may initially need to increase expenditures to realise future cost savings; for example, employing therapists and renting offices to provide CBT sessions. Third, not all patients with TRS who are eligible for clozapine would be willing to initiate clozapine. However, there is a lack of evidence about the acceptance rate of clozapine in clozapine-naive patients. Therefore, the original acceptance rate of clozapine was assumed to be $100 \%$ in the model. Finally, the study used to inform duration of delay in initiation of clozapine was based on a cohort of patients commencing clozapine in London between 2006 and 2010, ${ }^{4}$ because no better evidence was identified. To our knowledge, there is a lack of evidence about the duration of delay in initiating clozapine for patients with TRS in other parts of the UK, and so the generalisability of the duration of delay data used in our study is unknown. 
Huajie Jin (D), PhD, King's Health Economics, Institute of Psychiatry, Psychology \& Neuroscience, King's College London, UK; Paul Tappenden, PhD, Health Economics and Decision Science, School of Health and Related Research, University of Sheffield, UK James H. MacCabe (D), FRCPsych, Institute of Psychiatry, Psychology \& Neuroscience, King's College London, UK; Stewart Robinson, PhD, School of Business and Economics, Loughborough University, UK; Paul McCrone, PhD, Faculty of Education, Health and Human Sciences, University of Greenwich, UK S Sarah Byford (D, PhD, King's Health Economics, Institute of Psychiatry, Psychology \& Neuroscience, King's College London, UK

Correspondence: Huajie Jin. Email: huajie.jin@kcl.ac.uk

First received 27 Mar 2020, final revision 24 Oct 2020, accepted 4 Nov 2020

\section{Supplementary material}

To view supplementary material for this article, please visit https://doi.org/10.1192/bjp.2020 241.

\section{Data availability}

All data generated or analysed during this study are included in this published article and a previous publication about the methods of developing the schizophrenia whole-disease model.

\section{Author contributions}

H.J. conducted the economic analysis, including adapting the existing schizophrenia for this study, searching for and preparing input data, running the computer model and analysing the results. J.H.M., P.T., S.R. and S.B. advised on the overall study design and analysis. J.H.M. and P.M. helped with identification and selection of input data, and results interpretation. H.J. led the writing of the paper. All authors edited the paper and approved the final version. $H . J$ will serve as a guarantor for the overall content of the manuscript.

\section{Funding}

This work was supported by the Medical Research Council, UK (grant number MR/L011794/1 to H.J., J.H.M. and P.M.)

\section{Declaration of interest}

J.H.M. received grants from HS Lundbeck outside the submitted work. The sponsor and funder had no role in the design, data collection, analysis, interpretation, writing or publication of this study. The views expressed are those of the authors and not necessarily those of the Medical Research Council or HS Lundbeck. The other authors declare no competing interests.

ICMJE forms are in the supplementary material, available online at https://doi.org/10.1192/ bjp.2020.241.

\section{References}

1 National Institute for Health and Care Excellence (NICE). Psychosis and Schizophrenia in Adults: Prevention and Management. Clinical Guideline (CG178). NICE, 2014 (https://www.nice.org.uk/guidance/cg178).

2 NHS England. Report of the Early Intervention in Psychosis Audit. NHS England, 2016 (https://www.hqip.org.uk/resource/early-intervention-in-psychosisaudit-report/\#.X8eX2837SUk).

3 Clement S, Schauman O, Graham T, Maggioni F, Evans-Lacko S, Bezborodovs $\mathrm{N}$, et al. What is the impact of mental health-related stigma on help-seeking? A systematic review of quantitative and qualitative studies. Psychol Med 2015; 45(1): 11-27.

4 Howes OD, Vergunst F, Gee S, McGuire P, Kapur S, Taylor D. Adherence to treatment guidelines in clinical practice: study of antipsychotic treatment prior to clozapine initiation. Br J Psychiatry 2012; 201(6): 481-5.
5 Kashner TM, Rush AJ, Crismon ML, Toprac M, Carmody TJ, Miller AL, et al. An empirical analysis of cost outcomes of the Texas Medication Algorithm Project. Psychiatr Serv 2006; 57(5): 648-59.

6 Jin $\mathrm{H}$, Tappenden $\mathrm{P}$, Robinson S, Achilla E, MacCabe JH, Aceituno D, et al. A systematic review of economic models across the entire schizophrenia pathway. Pharmacoeconomics 2020; 38(6): 537-55.

7 Jin H, Tappenden P, MacCabe JH, Robinson S, Byford S. Evaluation of the costeffectiveness of services for schizophrenia in the UK across the entire care pathway in a single whole-disease model. JAMA Network Open 2020; 3(5): e205888-e.

8 Husereau D, Drummond M, Petrou S, Carswell C, Moher D, Greenberg D, et al. Consolidated Health Economic Evaluation Reporting Standards (CHEERS) statement. Pharmacoeconomics 2013; 31(5): 361-7.

9 Whitehead SJ, Ali S. Health outcomes in economic evaluation: the QALY and utilities. Br Med Bull 2010; 96(1): 5-21.

10 National Institute for Health and Care Excellence (NICE). Developing NICE Guidelines: The Manual. NICE, 2014 (https://www.nice.org.uk/media/default/ about/what-we-do/our-programmes/developing-nice-guidelines-the-manual. pdf).

11 Thien K, O'Donoghue B. Delays and barriers to the commencement of clozapine in eligible people with a psychotic disorder: a literature review. Early Interv Psychiatry 2019; 13(1): 18-23.

12 Najim H, Heath D, Singh P. Antipsychotic prescribing before clozapine in a community psychiatric hospital: a case note review. Psychiatr Danub 2013; 25 (suppl 2): S165-70.

13 Hutton P, Taylor PJ. Cognitive behavioural therapy for psychosis prevention: a systematic review and meta-analysis. Psychol Med 2014; 44(3): 449-68.

14 Samara MT, Dold M, Gianatsi M, Nikolakopoulou A, Helfer B, Salanti G, et al. Efficacy, acceptability, and tolerability of antipsychotics in treatment-resistant schizophrenia: a network meta-analysis. JAMA Psychiatry 2016; 73(3): 199210.

15 Fusar-Poli P, Rocchetti M, Sardella A, Avila A, Brandizzi M, Caverzasi E, et al Disorder, not just state of risk: meta-analysis of functioning and quality of life in people at high risk of psychosis. Br J Psychiatry 2015; 207(3): 198-206.

16 Alshowkan A, Curtis J, White Y. Quality of life for people with schizophrenia: a literature review. Arab J Psychiatry 2012; 23(2): 122-31.

17 National Institute for Health and Care Excellence (NICE). Psychosis and Schizophrenia in Adults. NICE Guideline (CG178). NICE, 2014 (https://www. nice.org.uk/guidance/cg178/evidence/full-guideline-490503565).

18 Curtis L. Unit Costs of Health \& Social Care 2017. Personal Social Services Research Unit, University of Kent, 2017 (https://www.pssru.ac.uk/projectpages/unit-costs/unit-costs-2017/)

19 National Collaborating Centre for Nursing and Supportive Care. Violence: The Short-Term Management of Disturbed/Violent Behaviour in In-Patient Psychiatric Settings and Emergency Departments. Royal College of Nursing (UK), 2005

20 Department of Health. NHS Reference Costs 2016 to 2017. Department of Health, 2017 (https://improvement.nhs.uk/resources/reference-costs/)

21 Kennedy JL, Altar CA, Taylor DL, Degtiar I, Hornberger JC. The social and economic burden of treatment-resistant schizophrenia: a systematic literature review. Int Clin Psychopharmacol 2014; 29(2): 63-76.

22 Centre for Economic Performance's Mental Health Policy Group. How Mental IIIness Loses Out in the NHS. London School of Economics and Political Science, 2012 (http://eprints.Ise.ac.uk/44572/).

23 McNicoll A. Mental Health Trust Funding Down 8\% from 2010 Despite Coalition's Drive for Parity of Esteem. Campaign to Save Mental Health Services in Norfolk \& Suffolk, 2015 (https://www.communitycare.co.uk/2015/ 03/20/mental-health-trust-funding-8-since-2010-despite-coalitions-drive-parity-esteem/).

24 Docherty M, Thornicroft G. Specialist mental health services in England in 2014: overview of funding, access and levels of care. Int J Ment Health Syst 2015; 9(1): 34

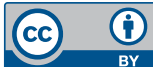

